\title{
A Probabilistic Analysis of the Power of Arithmetic Filters*
}

\author{
O. Devillers ${ }^{1}$ and F. P. Preparata ${ }^{2}$ \\ ${ }^{1}$ INRIA, BP 93, 06902 Sophia Antipolis, France \\ Olivier.Devillers@sophia.inria.fr \\ ${ }^{2}$ Department of Computer Science, Brown University, \\ Providence, RI 02912-1910, USA \\ franco@cs.brown.edu
}

\begin{abstract}
The assumption of real-number arithmetic, which is at the basis of conventional geometric algorithms, has been seriously challenged in recent years, since digital computers do not exhibit such capability. A geometric predicate usually consists of evaluating the sign of some algebraic expression. In most cases, rounded computations yield a reliable result, but sometimes rounded arithmetic introduces errors which may invalidate the algorithms. The rounded arithmetic may produce an incorrect result only if the exact absolute value of the algebraic expression is smaller than some (small) $\varepsilon$, which represents the largest error that may arise in the evaluation of the expression. The threshold $\varepsilon$ depends on the structure of the expression and on the adopted computer arithmetic, assuming that the input operands are error-free. A pair (arithmetic engine, threshold) is an arithmetic filter. In this paper we develop a general technique for assessing the efficacy of an arithmetic filter. The analysis consists of evaluating both the threshold and the probability of failure of the filter. To exemplify the approach, under the assumption that the input points be chosen randomly in a unit ball or unit cube with uniform density, we analyze the two important predicates "which-side" and "insphere." We show that the probability that the absolute values of the corresponding determinants be no larger than some positive value $V$, with emphasis on small $V$, is $\Theta(V)$ for the which-side predicate, while for the insphere predicate it is $\Theta\left(V^{2 / 3}\right)$ in dimension $1, O\left(V^{1 / 2}\right)$ in dimension 2, and $O\left(V^{1 / 2} \ln (1 / V)\right)$ in higher dimensions. Constants are small, and are given in the paper.
\end{abstract}

\footnotetext{
* This work was partially supported by ESPRIT LTR 21957 (CGAL) and by the U.S. Army Research Office under Grant DAAH04-96-1-0013. This work was done in part while O. Devillers was visiting Brown University.
} 


\section{Introduction}

The original model of Computational Geometry rests on real-number arithmetic, and under this assumption the issue of precision is irrelevant. However, the reality that computer calculations have finite precision has raised an increasing awareness of its effect on the quality and even the validity of geometric algorithms conceived within the original model, in the sense that algorithm correctness does not automatically translate into program correctness. In recent years this issue has been amply debated in the literature (see, e.g., $\left[\mathrm{BKM}^{+}\right],[\mathrm{FV}]$, and [Y]). In particular, it has been observed that while some degree of approximation may be tolerated in geometric constructions, the evaluation of predicates (the "tests" carried out in the execution of programs—-such as which-side, incircle, insphere) must be exact to ensure the structural (topological) correctness of the results [BMS], [Y], [LPT].

In principle, error-free predicate evaluation is achievable for error-free input operands, if the latter are treated as integers and the arithmetic is carried out with whatever operand length is required to express the intermediate results. Such a safe approach, however, if adopted in its crudest form, would involve enormous overheads and would be nearly impracticable, since the execution time of some operation (such as multiplication) may increase quadratically with the length of the representation.

As a time-saving alternative to exact arithmetic, it has been customary to resort to rounded (approximate) arithmetic (for example, floating-point arithmetic). Such practice can be modeled as follows. Evaluation of a predicate $P$ typically involves computing the value $\mu$ of some expression, built using rational operations. The value of $\mu$ can be mapped to one of three values: positive, zero, negative (referred to here as the "sign" of $\mu$ ), which defines the predicate $P$. Let $\mathcal{E}$ denote an evaluator for $P$, and let $\mu(\mathcal{E})$ denote the numerical value computed by $\mathcal{E}$. In general, $\mathcal{E}$ is an approximate evaluator of $\mu$, so that its use involves the adoption of a device, called a certifier, intended to validate the correctness of the evaluation. The pair (evaluator, certifier) is what has been referred to as a filter in the literature [FV], [MN]. Typically, the certifier for $\mathcal{E}$ compares $|\mu(\mathcal{E})|$ with a fixed threshold $\varepsilon(\mathcal{E}) \geq 0$. If $|\mu(\mathcal{E})| \geq \varepsilon(\mathcal{E})$, then the sign of $\mu(\mathcal{E})$ is reliable. Otherwise, the certifier is unable to validate the result and we have a failure of the filter. In such an event recourse to a more powerful filter is in order. This suggests the need to develop a family of filters of increasing precision (and complexity), to be used in sequence until failure no longer occurs. The last item of this sequence is the exact evaluator, for which the certifier is vacuous (i.e., $\varepsilon(\mathcal{E})=0$ ). Such an approach, with an obvious tradeoff between efficacy and efficiency, embodies the notion of adaptive precision.

From a practical standpoint it is therefore very important to gauge the efficacy of very simple filters, that is, their probability of success. If it turns out that if a filter has a high probability of success, then recourse to a more time-consuming filter (or exact computations) will be a rather rare event. Of course, any such estimate of efficacy rests on some arbitrary hypotheses on the a priori probability of problem instances. This is an important caveat; however, under reasonable hypotheses (uniform distributions), we submit that the estimates obtained will be a significant contribution to the assessment of the validity of such approaches.

In this paper we analyze two specific predicates, "which-side" and "insphere." Both predicates consist of computing the signs of appropriate $\delta \times \delta$ determinants, whose entries 
are specified with a fixed number of bits. Depending upon the adopted evaluation scheme (the choice of an equivalent expression for a given function) and upon the precision of the input operands (for example, only a fixed-length prefix of their representation may be used in the evaluation), only a prefix of the computed value is reliable. This means that if the absolute value of the determinant is above a known threshold $\varepsilon$, then its sign is also reliable.

Our objective is therefore twofold:

1. To compute the value of the threshold $\varepsilon$ for a given determinantal evaluation technique.

2. To compute the probability that the absolute value of the result of the evaluation does not exceed $\varepsilon$, i.e., the probability of filter failure.

We recall that when evaluating the determinant of a $\delta \times \delta$ matrix $A$ (a $\delta$-determinant, for short) we are computing the signed measure of a hyperparallelepiped defined by the $\delta$ vectors corresponding to the rows of $A$. Since each of the components of these vectors is an integer in the range $\left[-2^{b-1}, 2^{b-1}-1\right]$, a generic vector is (applied to the origin and) defined by its free terminus at grid points in a $\delta$-dimensional cube $\mathcal{C}_{\delta}$ of sidelength $2^{b}$ centered at the origin.

Our probability model assumes that all grid points within $\mathcal{C}_{\delta}$ have identical probability. Our analysis aims at estimating (a majorization of) the distribution of the volume of the hyperparallelogram described above. To obtain the desired result, we introduce some simplifications consistent with the objective to majorize the probability. Specifically, while our assumption is a discrete uniform distribution within $\mathcal{C}_{\delta}$, we begin by considering a continuous uniform distribution within the ball $\mathcal{B}_{\delta}$, the $\delta$-dimensional ball of radius 1 . The results obtained are then used to bound from above the distribution of the volume for uniform density within the cube $\mathcal{C}_{\delta}$ (the $\delta$-fold cartesian product of interval $[-1,1]$ ), and are finally extended to the target case of uniform discrete distribution. We shall recognize that the initial simplification (uniform density in $\mathcal{C}_{\delta}$ ) closely approximates the more realistic situation.

The paper is organized as follows. We begin with the "which-side" predicate (referred to as "determinant"), i.e., in Section 2 we carry out the probabilistic analysis in $\mathcal{B}_{\delta}$, for $\delta=1,2,3$ and arbitrary $\delta$ (detailed considerations of the low-dimensional cases has an obvious pedagogical motivation). In Section 3 we extend the results from the continuous ball to the discrete cube. In Section 4 we carry out an analogous analysis for the "insphere" predicate, which illustrates the adverse effect of dependencies among the determinant entries. Finally, in the Appendix we evaluate the precision of determinant evaluation by recursive expansion, and illustrate the efficacy/efficiency tradeoff.

\section{Probabilistic Analysis Within the Unit Ball}

Throughout this section we adopt the following notation: We let $x_{1}, x_{2}, \ldots, x_{\delta}$ be the coordinates of $\delta$-space, and let $p_{1}, p_{2}, \ldots, p_{\delta}$ be $\delta$ points in the unit ball of dimension $\delta$. We also denote by $\left|p_{1}, p_{2}, \ldots, p_{\delta}\right|$ the absolute value of the determinant defined by points $p_{1}, p_{2}, \ldots, p_{\delta}$. This quantity, which is the volume of the hyperparallelogram defined by the origin and by points $p_{1}, p_{2}, \ldots, p_{\delta}$, will also be denoted $a_{\delta}$. 
We begin by examining in some detail the cases of low-dimension determinants. Since the analysis is done in a visualizable geometric setting $(\delta \leq 3)$, it is preparatory to the more abstract higher-dimensional cases.

\subsection{1- and 2-Determinant}

Obviously, if $p_{1}$ is uniformly distributed between -1 and 1 , then

$$
\operatorname{Prob}\left(\left|p_{1}\right| \leq R\right)=R \text {. }
$$

Less trivial is the analysis of the two-dimensional case. We study the probability for $\left|p_{1}, p_{2}\right|$ to be smaller than a constant $A$ when $p_{1}$ and $p_{2}$ are distributed uniformly in the unit disk.

Once $p_{1}$ is chosen (due to the circular symmetry, $p_{1}$ is represented by a single parameter $a_{1}$, its distance from the origin), $p_{2}$ will yield an area between $a_{2}$ and $a_{2}+d a_{2}$ if it belongs to one of the two strips of width $d a_{2} / a_{1}$ depicted in Fig. 1 .

We then have

$$
\operatorname{Prob}\left(\left|p_{1}, p_{2}\right| \leq A\right)=\int_{0}^{1} \operatorname{Prob}\left(\left|p_{1}, p_{2}\right| \leq A \mid a_{1}\right) p\left(a_{1}\right) d a_{1} .
$$

Since the density function of $a_{1}$ is $p\left(a_{1}\right)=2 \pi a_{1} / \pi=2 a_{1}$, and the density of $a_{2}$ conditional on $a_{1}$ is $p\left(a_{2} \mid a_{1}\right) d a_{2}=(1 / \pi) 4 \sqrt{1-\left(a_{2} / a_{1}\right)^{2}}\left(d a_{2} / a_{1}\right)((1 / \pi)$ is the density in the unit disk, and $4 \sqrt{1-\left(a_{2} / a_{1}\right)^{2}}$ is the total length of the two strips) we have

$$
\begin{aligned}
\operatorname{Prob}\left(\left|p_{1}, p_{2}\right| \leq A\right) & =\int_{a_{1}=0}^{1} \int_{a_{2}=0}^{\min \left(A, a_{1}\right)} 2 p\left(a_{2} \mid a_{1}\right) a_{1} d a_{1} d a_{2} \\
& =\int_{0}^{A}\left(\int_{a_{2}}^{1} \frac{4}{\pi a_{1}} \sqrt{1-\left(\frac{a_{2}}{a_{1}}\right)^{2}} \cdot 2 a_{1} d a_{1}\right) d a_{2} \\
& =\int_{0}^{A} \frac{8}{\pi} d a_{2}\left|\sqrt{a_{1}^{2}-a_{2}^{2}}-a_{2} \arccos \frac{a_{2}}{a_{1}}\right|_{a_{2}}^{1} \\
& =\int_{0}^{A} \frac{8}{\pi} d a_{2}\left(\sqrt{1-a_{2}^{2}}-a_{2} \arccos a_{2}\right) \\
& =\frac{8}{\pi}\left|\frac{3}{4} a_{2} \sqrt{1-a_{2}^{2}}+\frac{1}{4} \arcsin a_{2}-\frac{1}{2} a_{2}^{2} \arccos a_{2}\right|_{0}^{A} \\
& =\frac{6}{\pi} A \sqrt{1-A^{2}}+\frac{2}{\pi} \arcsin A-\frac{4}{\pi} A^{2} \arccos A .
\end{aligned}
$$

One can easily verify that the value of the last expression is 1 for $A=1$, which is the maximum attainable value for the area of the parallelogram.

\subsection{3-Determinant}

Again we assume that points are uniformly distributed in the unit ball, and compute the probability that the volume of the parallelepiped defined by $O, p_{1}, p_{2}$, and $p_{3}$ is smaller than a constant $V \geq 0$. 


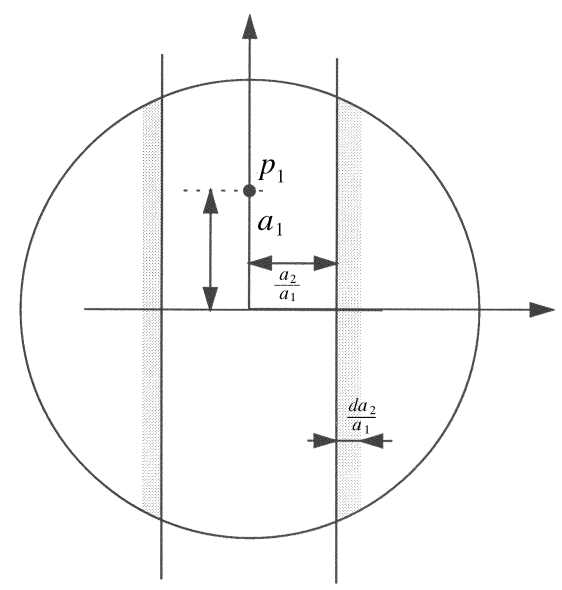

Fig. 1. For the analysis of the 2-determinant.

To compute this volume, we begin by considering the parallelogram defined by $O, p_{1}$, and $p_{2}$, evaluate its area, and then consider the distance of $p_{3}$ from the plane containing the parallelogram.

Distance $O p_{1}$ is between $a_{1}$ and $a_{1}+d a_{1}$ if $p_{1}$ belongs to a spherical crown of thickness $d a_{1}$ and area $4 \pi a_{1}^{2}$. Therefore the probability density of $a_{1}$ is $p\left(a_{1}\right)=(3 / 4 \pi) 4 \pi a_{1}^{2}=$ $3 a_{1}^{2}$ (note that $3 / 4 \pi$ is the density in the unit sphere).

Once $p_{1}$ has been chosen, the area of the parallelogram defined by $O, p_{1}$, and $p_{2}$ is between $a_{2}$ and $a_{2}+d a_{2}$ if $p_{2}$ belongs to the crown of thickness $d a_{2} / a_{1}$ of a cylinder of radius $a_{2} / a_{1}$ whose axis contains $O p_{1}$ (see Fig. 2(a)). Therefore the distribution of $a_{2}$
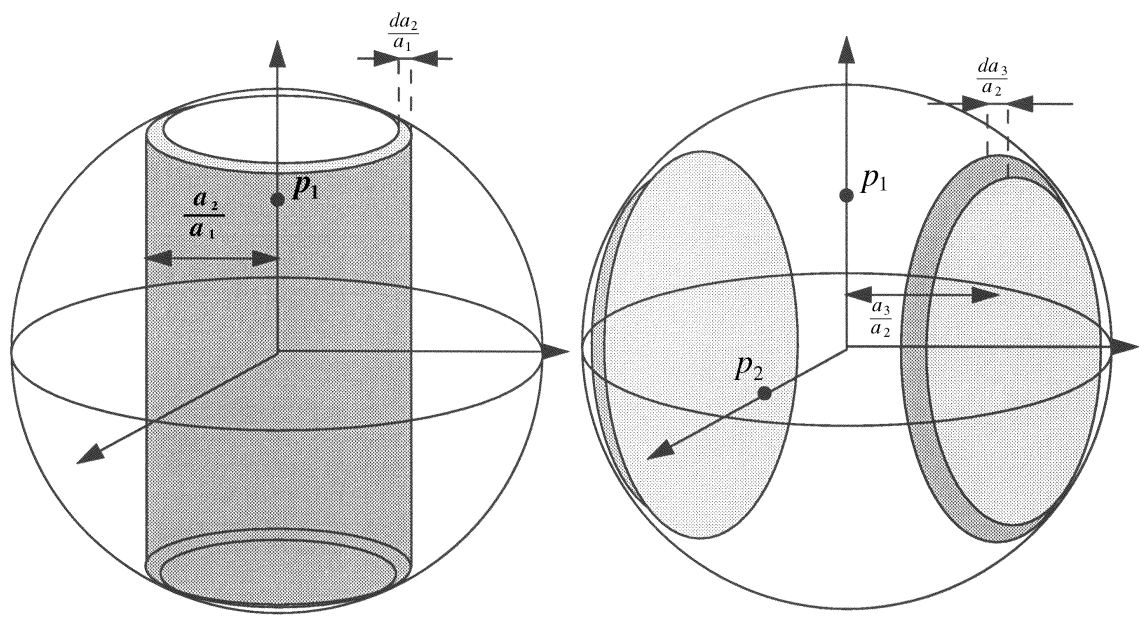

Fig. 2. For the analysis of the 3-determinant. 
conditional on $a_{1}$ is given by

$$
p\left(a_{2} \mid a_{1}\right) d a_{2}=\frac{3}{4 \pi} \cdot 2 \pi \frac{a_{2}}{a_{1}} \cdot 2 \sqrt{1-\left(\frac{a_{2}}{a_{1}}\right)^{2}} \cdot \frac{d a_{2}}{a_{1}}=\frac{3 a_{2}}{a_{1}^{2}} \sqrt{1-\left(\frac{a_{2}}{a_{1}}\right)^{2}} d a_{2} .
$$

Finally, once $p_{1}$ and $p_{2}$ have been chosen, the volume of the parallelepiped is between $a_{3}$ and $a_{3}+d a_{3}$ if $p_{3}$ belongs to one of the two spherical slices of width $d a_{3} / a_{2}$, parallel to the plane containing $O, p_{1}$, and $p_{2}$, and at distance $a_{3} / a_{2}$ from it (see Fig. 2(b)). Therefore the distribution of $a_{3}$ conditional on $a_{2}$ is given by

$$
p\left(a_{3} \mid a_{2}\right) d a_{3}=\frac{3}{4 \pi} \cdot 2 \cdot \pi\left(1-\left(\frac{a_{3}}{a_{2}}\right)^{2}\right) \cdot \frac{d a_{3}}{a_{2}}=\frac{3}{2 a_{2}}\left(1-\left(\frac{a_{3}}{a_{2}}\right)^{2}\right) d a_{3} .
$$

On the basis of this analysis, we can say

$$
\begin{aligned}
\operatorname{Prob}\left(\left|p_{1}, p_{2}, p_{3}\right| \leq V\right) & \int_{a_{1}=0}^{1} \int_{a_{2}=0}^{a_{1}} \int_{a_{3}=0}^{\min \left(V, a_{2}\right)} p\left(a_{1}\right) p\left(a_{2} \mid a_{1}\right) p\left(a_{3} \mid a_{2}\right) d a_{1} d a_{2} d a_{3} \\
= & \int_{0}^{V}\left(\int_{a_{3}}^{1}\left(\int_{a_{3}}^{a_{1}} p\left(a_{3} \mid a_{2}\right) p\left(a_{2} \mid a_{1}\right) p\left(a_{1}\right) d a_{2}\right) d a_{1}\right) d a_{3} \\
= & \int_{0}^{V} \frac{27}{2} d a_{3} \int_{a_{3}}^{1} d a_{1} \int_{a_{3}}^{a_{1}}\left(\frac{\sqrt{a_{1}^{2}-a_{2}^{2}}}{a_{1}}-a_{3}^{2} \frac{\sqrt{a_{1}^{2}-a_{2}^{2}}}{a_{1} a_{2}^{2}}\right) d a_{2} \\
= & \int_{0}^{V} \frac{27}{2} d a_{3} \int_{a_{3}}^{1} d a_{1}\left(\left|\frac{a_{2}}{2} \frac{\sqrt{a_{1}^{2}-a_{2}^{2}}}{a_{1}}+\frac{a_{1}}{2} \arcsin \frac{a_{2}}{a_{1}}\right|_{a_{3}}^{a_{1}}+a_{3}^{2}\left|\frac{\sqrt{a_{1}^{2}-a_{2}^{2}}}{a_{2} a_{1}}+\frac{1}{a_{1}} \arcsin \frac{a_{2}}{a_{1}}\right|_{a_{3}}^{a_{1}}\right) \\
= & \int_{0}^{V} \frac{27}{4} d a_{3} \int_{a_{3}}^{1} d a_{1}\left(\frac{\pi}{2} a_{1}+\pi \frac{a_{3}^{2}}{a_{1}}-\sqrt{a_{1}^{2}-a_{3}^{2}} \frac{3 a_{3}}{a_{1}}-a_{1} \arcsin \frac{a_{3}}{a_{1}}-2 \frac{a_{3}^{2}}{a_{1}} \arcsin \frac{a_{3}}{a_{1}}\right) \\
= & \int_{0}^{V} \frac{27}{4} d a_{3}\left(\frac{\pi}{4}-\pi a_{3}^{2} \ln a_{3}-\frac{7}{2} a_{3} \sqrt{1-a_{3}^{2}}+3 a_{3}^{2} \arccos a_{3}-\frac{1}{2} \arcsin a_{3}-2 a_{3}^{2} \int_{a_{3}}^{1} \frac{1}{a_{1}} \arcsin \frac{a_{3}}{a_{1}} d a_{1}\right) \\
= & \mid \frac{27 \pi}{16} a_{3}-\frac{9 \pi}{4} a_{3}^{3} \ln \left(a_{3}\right)+\frac{3 \pi}{4} a_{3}^{3}+\frac{63}{8}\left(\sqrt{1-a_{3}^{2}}\right)^{3}+\frac{27}{4} a_{3}^{3} \arccos \left(a_{3}\right)-\frac{27}{4} a_{3}^{2} \sqrt{1-a_{3}^{2}}-\frac{9}{2}\left(\sqrt{1-a_{3}^{2}}\right)^{3} \\
& -\frac{27}{8} a_{3} \arcsin \left(a_{3}\right)-\left.\frac{27}{8} \sqrt{1-a_{3}^{2}}\right|_{0} ^{V}-\frac{27}{2} \int_{0}^{V} \int_{a_{3}}^{1} \frac{a_{3}^{2}}{a_{1}} \arcsin \frac{a_{3}}{a_{1}} d a_{1} d a_{3} \\
= & \left|\frac{27 \pi}{16} a_{3}-\frac{9 \pi}{4} a_{3}^{3} \ln a_{3}+\frac{3 \pi}{4} a_{3}^{3}+\frac{27}{4} a_{3}^{3} \arccos a_{3}-\frac{81}{8} a_{3}^{2} \sqrt{1-a_{3}^{2}}-\frac{27}{8} a_{3} \arcsin a_{3}\right|_{0}^{V} \frac{a_{3}^{2}}{a_{1}} \arcsin \frac{a_{3}}{a_{1}} d a_{1} d a_{3} . \\
= & -\frac{27}{a_{3}} \int^{2} \\
= &
\end{aligned}
$$

The latter integral is not elementarily computable. Since the integrand is always positive, so is the integral. To neglect it corresponds to majorizing the probability, which is conservative for our analysis. Therefore we write

$\operatorname{Prob}\left(\left|p_{1}, p_{2}, p_{3}\right| \leq V\right) \leq \frac{27 \pi}{16} V-\frac{9 \pi}{4} V^{3} \ln V+\frac{3 \pi}{4} V^{3}-\frac{81}{8} V^{2} \sqrt{1-V^{2}}+\frac{27}{4} V^{3} \arccos V-\frac{27}{8} V \arcsin V$. 
If we set $V=1$, then the neglected term can be evaluated exactly to $3 \pi / 4-1$ by exchanging the order of integration, and we correctly obtain the value 1 for the probability.

Furthermore, using the inequalities $a_{3} \leq a_{1}$ and $\arcsin a_{3} / a_{1} \leq \pi / 2$, the neglected term can be bounded from above as $\frac{27}{2} \int_{0}^{V} \int_{a_{3}}^{1} a_{3}(\pi / 2) d a_{1} d a_{3} \leq(27 \pi / 8) V^{2}$, so we guarantee the tightness of the approximation of the probability by $(27 \pi / 16) V$ when $V$ is small.

The preceding analysis, in its simplicity, reveals the essential items for the evaluation of the relevant conditional probability densities. Specifically, referring concretely to the case $\delta=3$, due to the assumption of uniform distribution of the points in the unit sphere, the conditional probability density $p\left(a_{i} \mid a_{i-1}\right) d a_{i}$ of $a_{i}$ given $a_{i-1}, i=2,3$, is proportional (through the value of the density in the unit sphere) to the volume of some three-dimensional domain. The latter is a thin crown (of thickness $d a_{i} / a_{i-1}$ ) of a threedimensional surface $\mathcal{S}_{3, i}$ which is the locus of the points at a distance between $a_{i} / a_{i-1}$ and $a_{i} / a_{i-1}+d a_{i} / a_{i-1}$ from the flat $\mathcal{F}_{i-1}$ spanned by variables $x_{1}, \ldots, x_{i-1}$. Surface $\mathcal{S}_{3, i}$ has a very simple structure. Let $(u, v)$ be a pair of points realizing the distance $a_{i} / a_{i-1}$, with $u \in \mathcal{F}_{i-1}$ and $v \in \mathcal{S}_{3, i}$. Point $v$ belongs to the boundary of a $(4-i)$ dimensional ball of radius $a_{i} / a_{i-1}$ of which point $u$ is the center: therefore this entire boundary belongs to $\mathcal{S}_{3, i}$ (in the discussion above, this boundary consists of a circle for $i=2$ and of two points for $i=3$ ). Moreover, since $u$ belongs to a flat $\mathcal{F}_{i-1}$, any translate of $v$ within the unit sphere in a flat parallel to $\mathcal{F}_{i-1}$ also belongs to $\mathcal{S}_{3, i}$. These translates form the intersection of the unit sphere with a flat at distance $a_{i} / a_{i-1}$ from the center of the sphere, and therefore are an $(i-1)$-dimensional ball of radius $\sqrt{1-\left(a_{1} / a_{i-1}\right)^{2}}$ (in the discussion above this ball consists of a segment for $i=2$ and of a disk for $i=3$ ). We conclude that $\mathcal{S}_{3, i}$ is the cartesian product of the boundary of a $(4-i)$-dimensional ball of radius $a_{i} / a_{i-1}$ (the "boundary" term) and of an $(i-1)$-dimensional ball of radius $\sqrt{1-\left(a_{1} / a_{i-1}\right)^{2}}$ (the "domain" term). The expression for the conditional probability density consists of four factors: the density within the unit sphere, the measure of the boundary term, the measure of the domain term, and the thickness of the crown. These four items (in the order given) are evidenced in (a) and (b).

\subsection{Higher-Dimensional Determinant}

We now extend the preceding analysis to arbitrary dimension $\delta$. If we assume for a onedimensional volume (a distance) the conventional degree of 1 , then the volume and the surface of a $j$-dimensional domain have respective degrees $j$ and $j-1$. Let $v_{j}(r)$ and $s_{j}(r)$ respectively denote volume and surface of a $j$-dimensional ball of radius $r$. We recall that $[\mathrm{B}, 9.12 .4 .6]$

$$
v_{i}(r)=\frac{\pi^{i / 2}}{(i / 2) !} r^{i} \quad \text { for } i \text { even, } \quad v_{i}(r)=\frac{2^{i} \pi^{(i-1) / 2}((i-1) / 2) !}{i !} r^{i} \quad \text { for } i \text { odd } .
$$

The probability density $p\left(a_{1}\right) d a_{1}=\operatorname{Prob}\left(a_{1} \leq\left|O p_{1}\right| \leq a_{1}+d a_{1}\right)$ is obviously given by $\left(s_{\delta}\left(a_{1}\right) / v_{\delta}(1)\right) d a_{1}$. Referring next to the observations at the end of the preceding subsection, the conditional probability density $p\left(a_{i} \mid a_{i-1}\right) d a_{i}$ of $a_{i}$ after $p_{1}, p_{2}, \ldots, p_{i-1}$ have been chosen (conventionally in the flat described by coordinates $x_{1}, x_{2}, \ldots, x_{i-1}$ ) 
to realize the value $a_{i-1}$, has the following expression:

$$
p\left(a_{i} \mid a_{i-1}\right) d a_{i}=\frac{1}{v_{\delta}(1)} \cdot s_{\delta-i+1}\left(\frac{a_{i}}{a_{i-1}}\right) \cdot v_{i-1}\left(\sqrt{1-\left(\frac{a_{i}}{a_{i-1}}\right)^{2}}\right) \cdot \frac{d a_{i}}{a_{i-1}} .
$$

Therefore, since $v_{i}(r)=v_{i}(1) \cdot r^{i}$ and $s_{i}(r)=i \cdot v_{i}(1) \cdot r^{i-1}$, we have

$$
\begin{aligned}
p\left(a_{1}\right) \prod_{i=2}^{\delta} p\left(a_{i} \mid a_{i-1}\right) d a_{i}= & \frac{\delta v_{\delta}(1) a_{1}^{\delta-1}}{v_{\delta}(1)} \prod_{i=2}^{\delta} \frac{1}{v_{\delta}(1)} \cdot(\delta-i+1) v_{\delta-i+1}(1) \\
& \cdot\left(\frac{a_{i}}{a_{i-1}}\right)^{\delta-i} \cdot v_{i-1}(1) \cdot\left(\sqrt{1-\left(\frac{a_{i}}{a_{i-1}}\right)^{2}}\right)^{i-1} \cdot \frac{d a_{i}}{a_{i-1}} \\
= & \frac{\delta ! a_{1}^{\delta-1}}{v_{\delta}(1)^{\delta-1}}\left(\prod_{i=2}^{\delta} v_{\delta-i+1}(1) v_{i-1}(1)\right) \\
& \times\left(\prod_{i=2}^{\delta} \frac{a_{i}^{\delta-i}}{a_{i-1}^{\delta-i+1}}\left(\sqrt{1-\left(\frac{a_{i}}{a_{i-1}}\right)^{2}}\right)^{i-1} d a_{i}\right) .
\end{aligned}
$$

In the rightmost term above the product of the powers of the $a_{i}$ 's simplifies to $1 / a_{1}^{\delta-1}$, so that

$$
=\frac{\delta !\left(\prod_{i=1}^{\delta-1} v_{i}(1)\right)^{2}}{v_{\delta}(1)^{\delta-1}} \prod_{i=2}^{\delta}\left(\sqrt{1-\left(\frac{a_{i}}{a_{i-1}}\right)^{2}}\right)^{i-1} d a_{i}
$$

The last expression contains a constant depending only on $\delta$, which will be denoted

$$
k_{\delta} \triangleq \frac{\delta !\left(\prod_{i=1}^{\delta-1} v_{i}(1)\right)^{2}}{v_{\delta}(1)^{\delta-1}} .
$$

Now we can write the probability for the absolute value of the determinant to be no larger than $V$ :

$$
\begin{aligned}
\operatorname{Prob}\left(\left|p_{1}, p_{2}, \ldots, p_{\delta}\right| \leq V\right)= & k_{\delta} \int_{a_{1}=0}^{1} \int_{a_{2}=0}^{a_{1}} \ldots \int_{a_{\delta-1}=0}^{a_{\delta-2}} \int_{a_{\delta}=0}^{\min \left(V, a_{\delta-1}\right)} \prod_{i=2}^{\delta}\left(\sqrt{1-\left(\frac{a_{i}}{a_{i-1}}\right)^{2}}\right)^{i-1} d a_{i} \\
= & k_{\delta} \int_{a_{1}=0}^{1} \int_{a_{2}=0}^{a_{1}} \ldots \int_{a_{\delta-1}=0}^{a_{\delta-2}}\left(\int_{a_{\delta}=0}^{\min \left(V, a_{\delta-1}\right)}\left(\sqrt{1-\left(\frac{a_{\delta}}{a_{\delta-1}}\right)^{2}}\right)^{i-1} d a_{\delta}\right) \\
& \times \prod_{i=2}^{\delta-1}\left(\sqrt{1-\left(\frac{a_{i}}{a_{i-1}}\right)^{2}}\right)^{i-1} d a_{i} .
\end{aligned}
$$

We now observe that the integral

$$
\int_{a_{\delta}=0}^{\min \left(V, a_{\delta-1}\right)}\left(\sqrt{1-\left(\frac{a_{\delta}}{a_{\delta-1}}\right)^{2}}\right)^{i-1} d a_{\delta}
$$


is trivially bounded by $V$. Therefore we obtain the following upper bound:

$$
\operatorname{Prob}\left(\left|p_{1}, p_{2}, \ldots, p_{\delta}\right| \leq V\right) \leq k_{\delta} V \int_{0}^{1} \ldots \int_{0}^{a_{\delta-2}} \prod_{i=2}^{\delta-1}\left(\sqrt{1-\left(\frac{a_{i}}{a_{i-1}}\right)^{2}}\right)^{i-1} d a_{i}
$$

Since the latter integral does not depends on $V$, its value is another constant, which we denote $\mathcal{I}_{\delta}$ and which depends only on $\delta$. However, when $V=1$, (c) yields $\operatorname{Prob}\left(\left|p_{1}, p_{2}, \ldots, p_{\delta}\right| \leq 1\right)=k_{\delta} \mathcal{I}_{\delta+1}$ and, since $\operatorname{Prob}\left(\left|p_{1}, p_{2}, \ldots, p_{\delta}\right| \leq 1\right)=1$, we get $\mathcal{I}_{\delta}=1 / k_{\delta-1}$.

Finally, we conclude

$$
\begin{aligned}
\operatorname{Prob}\left(\left|p_{1}, p_{2}, \ldots, p_{\delta}\right| \leq V\right) & \leq k_{\delta} \mathcal{I}_{\delta} V \\
& \leq \delta \frac{v_{\delta-1}(1)^{\delta}}{v_{\delta}(1)^{\delta-1}} V \triangleq \sigma_{\delta} V .
\end{aligned}
$$

For small $\delta$, the bounds are given below. For $\delta=1,2,3$ the bounds coincide with the values previously found, that is,

$$
\begin{aligned}
& \sigma_{1}=1, \\
& \sigma_{2}=\frac{2^{3}}{\pi} \approx 2.5, \\
& \sigma_{3}=\frac{3^{3} \pi}{2^{4}} \approx 5.3, \\
& \sigma_{4}=\frac{2^{13}}{3^{4} \pi^{2}} \approx 10, \\
& \sigma_{5}=\frac{3^{4} 5^{5} \pi^{2}}{2^{17}} \approx 19, \\
& \sigma_{6}=\frac{2^{24}}{5^{6} \pi^{3}} \approx 35 .
\end{aligned}
$$

\section{From Continuous Ball to Discrete Cube}

\subsection{From Continuous Ball to Continuous Cube}

The above calculations have been carried out for points uniformly distributed inside the $\delta$-dimensional ball of radius 1 , referred to as $\mathcal{B}_{\delta}$. This assumption may not seem to model the real situation for two reasons: (i) points manipulated by computers have discrete rather than continuous coordinates, and (ii) points are more reasonably assumed to be uniformly distributed in a cube than in a ball (as in the case when each coordinate is independently and uniformly selected).

We first show that the previous result relative to the ball $\mathcal{B}_{\delta}$ induces a similar result for uniform density in the unit cube $\mathcal{C}_{\delta}=[-1,1]^{\delta}$.

Note that $\mathcal{C}_{\delta}$ is contained within a $\delta$-dimensional ball of radius $\sqrt{\delta}$, denoted $\sqrt{\delta} \mathcal{B}_{\delta}$. First, we consider points of $\mathcal{C}_{\delta}$ as points of $\sqrt{\delta} \mathcal{B}_{\delta}$ and apply a homothety with a factor 
$1 / \sqrt{\delta}$, thereby obtaining

$$
\begin{aligned}
\operatorname{Prob}\left(\left|p_{1}, p_{2}, \ldots, p_{\delta}\right|\right. & \left.\leq V \mid p_{i} \in \sqrt{\delta} \mathcal{B}_{\delta}\right) \\
& =\operatorname{Prob}\left(\left|p_{1}, p_{2}, \ldots, p_{\delta}\right| \leq \sqrt{\delta}^{-\delta} V \mid p_{i} \in \mathcal{B}_{\delta}\right) \leq \frac{\sigma_{\delta}}{\sqrt{\delta}^{\delta}} V .
\end{aligned}
$$

Next, we wish to restrict the points to belong to $\mathcal{C}_{\delta}$, i.e., we consider the event $p_{i} \in$ $\sqrt{\delta} \mathcal{B}_{\delta}, i=1, \ldots, \delta$, as the union of the event $p_{i} \in \mathcal{C}_{\delta}, i=1, \ldots, \delta$, and its negation. The probability of this event is clearly $\left(2^{\delta} / v_{\delta}(1) \sqrt{\delta}^{\delta}\right)^{\delta}$, so that

$$
\begin{aligned}
\operatorname{Prob}\left(\left|p_{1}, p_{2}, \ldots, p_{\delta}\right|\right. & \left.\leq V \mid p_{i} \in \sqrt{\delta} \mathcal{B}_{\delta}\right) \\
& \geq\left(\frac{2^{\delta}}{v_{\delta}(1) \sqrt{\delta}^{\delta}}\right)^{\delta} \operatorname{Prob}\left(\left|p_{1}, p_{2}, \ldots, p_{\delta}\right| \leq V \mid p_{i} \in \mathcal{C}_{\delta}\right)
\end{aligned}
$$

which gives us an upper bound to the probablity in question. Specifically

$$
\begin{aligned}
\operatorname{Prob}\left(\left|p_{1}, p_{2}, \ldots, p_{\delta}\right|\right. & \left.\leq V \mid p_{i} \in \mathcal{C}_{\delta}\right) \\
& \leq\left(\frac{v_{\delta}(1) \sqrt{\delta}}{2^{\delta}}\right)^{\delta} \frac{\sigma_{\delta}}{\sqrt{\delta}^{\delta}} V \leq \frac{\delta v_{\delta}(1) v_{\delta-1}(1)^{\delta} \sqrt{\delta}{ }^{\delta(\delta-1)}}{2^{\delta^{2}}} \triangleq \psi_{\delta} V .
\end{aligned}
$$

Practically, for small values of $\delta$ we get

$$
\begin{aligned}
& \psi_{1}=1 \\
& \psi_{2}=\pi \\
& \psi_{3}=\frac{27}{128} \pi^{4} \approx 21, \\
& \psi_{4}=\frac{32}{81} \pi^{6} \approx 380 \\
& \psi_{5}=\frac{9,765,625}{402,653,184} \pi^{12} \approx 23,000 \\
& \psi_{6}=\frac{19,683}{125,000} \pi^{15} \approx 4.5 \cdot 10^{6} .
\end{aligned}
$$

The previous computation can probably be generalized to other kinds of domains provided the ratio between the volumes of the inscribed ball and of the circumscribing ball is bounded.

\subsection{From Continuous Cube to Discrete Cube}

In this section we discuss why the results obtained for continuous density of points are still useful for discrete probability, i.e., when points belong to a regular grid of $1 / \eta^{\delta}$ points inside $\mathcal{C}_{\delta}$.

Notice that the preceding results are clearly incorrect for discrete probabilities. In fact, they prescribe $\operatorname{Prob}\left(\left|p_{1}, p_{2}, \ldots, p_{\delta}\right|=0\right)=0$, which is false for discrete probabilities. For example, in two dimensions, when $p_{1}$ is chosen, $p_{2}$ coincides with $p_{1}$ or with the origin with probability $2 \eta^{2}$, and yet the determinant value is 0 . For a less trivial case 
(when $p_{1}, p_{2}$, and the origin are distinct), the event $p_{1}=\left(x_{1}, x_{2}\right)$ and $p_{2}=\left(2 x_{1}, 2 x_{2}\right)$, with $x_{1}<\frac{1}{2}$ and $y_{1}<\frac{1}{2}$, has probability $\eta^{2} / 4>0$ while the determinant is still 0 . However, we can still prove that $\operatorname{Prob}\left(\left|p_{1}, \ldots, p_{\delta}\right| \leq V\right)=O(V)$ when $\eta$ is smaller than $V$.

If $p_{1}, \ldots, p_{\delta}$ is a set of points in $\mathcal{C}_{\delta}$ whose determinant is not larger than $V$, we map it to a the nearest set of grid points $p_{1}^{\prime}, \ldots, p_{\delta}^{\prime}$ whose determinant is not too large. More precisely, if $\mathcal{P}=\left(p_{1}, \ldots, p_{\delta}\right), \mathcal{P}^{\prime}=\left(p_{1}^{\prime}, \ldots, p_{\delta}^{\prime}\right)$, and $\mathcal{D}=\mathcal{P}^{\prime}-\mathcal{P}=\left(d_{1}, \ldots, d_{\delta}\right)$, we have

$$
\left|\mathcal{P}^{\prime}\right|=|\mathcal{P}+\mathcal{D}|=|\mathcal{P}|+\sum_{I \subset \mathbb{Z}_{\delta}, I \neq \emptyset}\left|\mathcal{P} \mathcal{D}_{I}\right|
$$

where $I$ is a nonempty subset of $\{1,2, \ldots, \delta\}$ and $\left|\mathcal{P} \mathcal{D}_{I}\right|$ is the determinant obtained by replacing, for each $i \in I, p_{i}$ with $d_{i}$ in $|\mathcal{P}|$. The above result follows from the multilinearity of the determinant. If the cardinality of $I$ is $j$, we can bound from above the absolute value of $\left|\mathcal{P} \mathcal{D}_{I}\right|$ by the product of the norms of its vector. Since $\left\|p_{i}\right\| \leq \sqrt{\delta}$ and $\left\|d_{i}\right\| \leq \sqrt{\delta}(\eta / 2)$ we have $\left|\mathcal{P} \mathcal{D}_{I}\right| \leq \sqrt{\delta}^{\delta}\left(\eta^{j} / 2^{j}\right)$. By grouping the $\left(\begin{array}{l}\delta \\ j\end{array}\right)$ terms with identical value of $j$ we get

$$
|| \mathcal{P}^{\prime}|-| \mathcal{P}|| \leq \sqrt{\delta}^{\delta}\left[\left(1+\frac{\eta}{2}\right)^{\delta}-1\right] \approx \delta \sqrt{\delta}^{\delta} \frac{\eta}{2} .
$$

Referring now to the $\delta^{\delta}$-dimensional space whose points are the sets $p_{1}, \ldots, p_{\delta}$, the determinant $\left|\mathcal{P}^{\prime}\right|$ is no larger than $V$ if all the points $\mathcal{P}$ in the hypervoxel (in $\delta^{\delta}$ dimensions) have determinant $|\mathcal{P}|$ no larger than $V+\delta \sqrt{\delta}^{\delta}(\eta / 2)$. Since, clearly, a random point $\mathcal{P}$ for the continuous distribution can be in any voxel with the same probability, we conclude that

$$
\begin{aligned}
\operatorname{Prob} & \left(\left|p_{1} \cdots p_{\delta}\right| \leq V \mid \underset{\text { distribution in }}{\text { discrete }} \mathcal{C}_{\delta}\right) \\
& \leq \operatorname{Prob}\left(\left|p_{1} \cdots p_{\delta}\right| \leq V+\delta \sqrt{\delta} \frac{\eta}{2} \mid \begin{array}{c}
\text { continuous } \\
\text { distribution in }
\end{array}\right. \\
\quad & \left.\mathcal{C}_{\delta}\right) \\
& \leq \psi_{\delta} \cdot\left(V+\delta \sqrt{\delta} \frac{\eta}{2}\right) .
\end{aligned}
$$

\section{The Insphere Test}

In the preceding analysis of the "which-side" predicate, the points defining the hyperparallelogram were assumed to be independent and equally distributed. In this section we consider a case for which there exist dependencies among the coordinates of the points: the "insphere" predicate. This predicate, referred to as $\delta$-insphere for short, tests whether in $\delta$ dimensions the origin lies inside the hypersphere defined by $(\delta+1)$ other arbitrary points $p_{i}=\left(x_{i, 1}, x_{i, 2}, \ldots, x_{i, \delta}\right), i=1,2, \ldots, \delta+1$.

It is well known that the $\delta$-dimensional insphere test is embodied in the sign of the determinant:

$$
\Delta_{\delta} \triangleq\left|\begin{array}{ccccc}
x_{11} & x_{12} & \cdots & x_{11}^{2}+x_{12}^{2}+\cdots+x_{1 \delta}^{2} & 1 \\
x_{21} & x_{22} & \cdots & x_{21}^{2}+x_{22}^{2}+\cdots+x_{2 \delta}^{2} & 1 \\
\cdots & & & & \\
x_{\delta+2,1} & x_{\delta+2,2} & \cdots & x_{\delta+2,1}^{2}+x_{\delta+2,2}^{2}+\cdots+x_{\delta+2, \delta}^{2} & 1
\end{array}\right| .
$$


Without loss of generality, one of these points $\left(p_{\delta+2}\right)$ can be chosen as the origin $O$, so that the above determinant becomes

$$
\Delta_{\delta}=\left|\begin{array}{cccc}
x_{11} & x_{12} & \cdots & x_{11}^{2}+x_{12}^{2}+\cdots+x_{1 \delta}^{2} \\
x_{21} & x_{22} & \cdots & x_{21}^{2}+x_{22}^{2}+\cdots+x_{2 \delta}^{2} \\
\cdots & & & \\
x_{\delta+1,1} & x_{\delta+1,2} & \cdots & x_{\delta+1,1}^{2}+x_{\delta+1,2}^{2}+\cdots+x_{\delta+1, \delta}^{2}
\end{array}\right| .
$$

These $\delta+1$ points are assumed to be evenly distributed in the unit cube $\mathcal{C}_{\delta}$.

\subsection{1-Insphere Test}

We can model the problem as follows. The origin $O$ and $u$ define the one-dimensional sphere; $v$ is the query point. Parameters $u$ and $v$ are independent and uniformly distributed in $[-1,1]$, and we wish to evaluate the probability of the following event:

$$
\left|\Delta_{1}\right| \leq A \quad \text { with } \quad \Delta_{1}=\left|\begin{array}{cc}
u & u^{2} \\
v & v^{2}
\end{array}\right|
$$

We can view the above determinant as defined by two points $(u, v)$ and $\left(u^{2}, v^{2}\right)$ in the $u, v$ plane. Clearly, the choice of point $(u, v)$ completely determines the determinant value. Point $(u, v)$ is uniformly distributed in the square $[-1,1] \times[-1,1]$. Since $u v(v-u)$ is the determinant value, the determinant is null on the three lines $u=0, v=0$, and $u=v$; its values are symmetric with respect to the line $u=-v$ and antisymmetric with respect to $u=v$. Therefore for any value of $0 \leq A \leq 2$ it is sufficient to evaluate the probability in the quadrant $-u \leq v \leq u, 0 \leq u \leq 1$ (fully shaded quadrant in Fig. 3) and multiply it by 4 . In the upper semiquadrant (where the determinant is negative) the contour lines have equations

$$
v=\frac{u}{2} \pm \frac{1}{2} \sqrt{u^{2}-4 \frac{A}{u}}
$$

The two curves for fixed $A$ join with a common vertical tangent at the point $(\mu, \mu / 2)$ where $\mu \triangleq(4 A)^{1 / 3}$ (notice that such curves exists only for $A<\frac{1}{4}$ ). In the lower semiquadrant (where the determinant is positive) contour lines have equations

$$
v=\frac{u}{2}-\frac{1}{2} \sqrt{u^{2}+4 \frac{A}{u}} \text { and } \quad v=-u
$$

and intersect the line $v=-u$ at point $(v,-v)$, where $v \triangleq \frac{1}{2}(4 A)^{1 / 3}=\frac{1}{2} \mu$. The probability of the event described above is given by the heavily shaded area in the Fig. 3 (in fact this area should be multiplied by 4 since there are four quadrants, and normalized, dividing by 4 , since 4 is the area of the square). The area is also 1 minus the area of the lightly shaded region. The latter area, which we want to bound from below is given by

$$
\mathcal{I} \triangleq \int_{\nu}^{1}\left(\frac{3}{2} u-\frac{u}{2} \sqrt{1+4 \frac{A}{u^{3}}}\right) d u+2 \int_{\mu}^{1} \frac{u}{2} \sqrt{1-4 \frac{A}{u^{3}}} d u .
$$




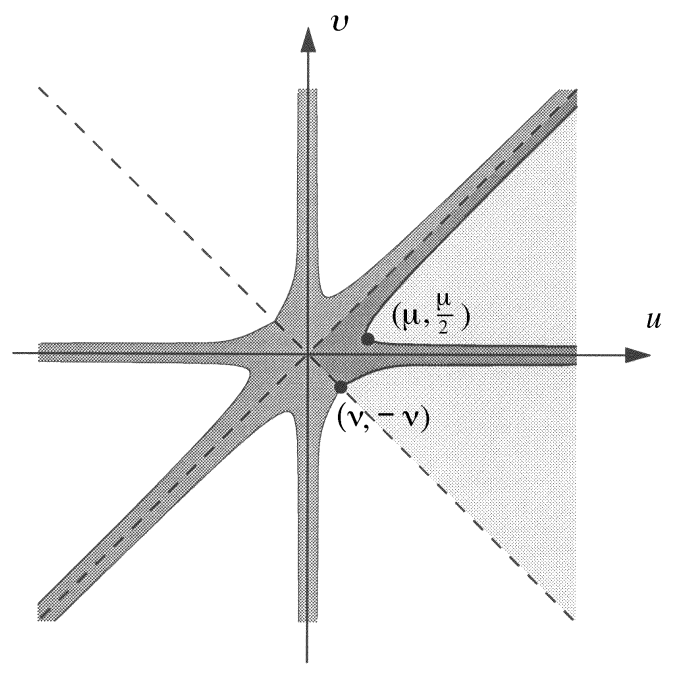

Fig. 3. The $(u, v)$-region defining $(|\Delta| \leq A)$ in the 1-insphere test.

We now observe that

$$
\frac{3}{2}-\frac{1}{2} \sqrt{1+4 \frac{A}{u^{3}}} \geq 1-\frac{A}{u^{3}}
$$

and (for $A \leq \frac{1}{4}$ )

$$
\sqrt{1-4 \frac{A}{u^{3}}} \geq 1-4 \frac{A}{u^{3}}
$$

so that

$$
\begin{aligned}
\mathcal{I} & \geq \int_{v}^{1} u d u-A \int_{v}^{1} \frac{1}{u^{2}} d u+\int_{\mu}^{1} u d u-4 A \int_{\mu}^{1} \frac{1}{u^{2}} d u \\
& =\frac{1}{2}-\frac{v^{2}}{2}+A-\frac{A}{v}+\frac{1}{2}-\frac{\mu^{2}}{2}+4 A-\frac{4 A}{\mu}
\end{aligned}
$$

If we now use the fact that $2 v=\mu=(4 A)^{1 / 3}$, then we obtain

$$
\operatorname{Prob}\left(\left|\Delta_{1}\right| \leq A\right) \leq \frac{17 \sqrt[3]{2}}{4} A^{2 / 3}-5 A \leq 5.355 A^{2 / 3} .
$$

A direct numerical calculation gives $\operatorname{Prob}\left(\left|\Delta_{1}\right| \leq \frac{1}{4}\right)=0.7$, while the value of the above bounding expression for the same value of $A$ is 0.85 (an excellent agreement considering the rather high value of $A$ ). For $A \geq \frac{1}{4}$, the lightly shaded region in the upper semiquadrant disappears yielding

$$
\operatorname{Prob}\left(\left|\Delta_{1}\right| \leq A\right) \leq 3 \sqrt[3]{2} A^{2 / 3}-4 A \leq 3.78 A^{2 / 3}
$$

but, considering the high range of $A$, this expression has little practical interest. 
Alternatively, we may consider the following formulation. Rather than lifting point $v$ to the parabola $y=v^{2} u-u^{2} v$ (for fixed positive $u$ ), we lift it to the parabola $y=$ $v^{2}-u v \triangleq F(v)$ (which intersects the $v$-axis at 0 and $u$ ) so that

$$
\operatorname{Prob}(|\Delta| \leq A)=\operatorname{Prob}\left(|F(v)| \leq \frac{A}{u}\right) .
$$

This formulation will be useful when considering the multidimensional case in Section 4.2 .2

\subsection{Higher-Dimensional Insphere Test}

By elementary column operations the determinant $\Delta_{\delta}$ can be transformed into one where the last column has zero entries except in the last row. Specifically, $\Delta_{\delta}=0$ defines the circumsphere $S$ of the points $O, p_{1}, \ldots, p_{\delta}$, whose center is the point $\left(c_{1} / 2, \ldots, c_{\delta} / 2\right)$. Subtracting column $i$ times $c_{i}(i=1, \ldots, \delta)$ from the last column, we obtain

$$
\Delta_{\delta}=\left|\begin{array}{ccccc}
x_{11} & x_{12} & \cdots & x_{1 \delta} & 0 \\
x_{21} & x_{22} & \cdots & x_{2 \delta} & 0 \\
\vdots & \vdots & & \vdots & \vdots \\
x_{\delta 1} & x_{\delta 2} & \cdots & x_{\delta \delta} & 0 \\
x_{\delta+1,1} & x_{\delta+1,2} & \cdots & x_{\delta+1, \delta} & W
\end{array}\right| .
$$

The determinant of the intersection of the first $\delta$ rows and columns of the above matrix gives the signed volume $v$ of the hyperparallelogram defined by the first $\delta$ points and $W$ has the dimension of the square of a length and is the value of the function

$$
F\left(x_{1}, \ldots, x_{\delta}\right)=x_{1}^{2}+x_{2}^{2}+\cdots+x_{\delta}^{2}-c_{1} x_{1}-\cdots-c_{\delta} x_{\delta}
$$

evaluated at point $p_{\delta+1}$. We let $u^{2} \triangleq \frac{1}{4}\left(c_{1}^{2}+\cdots+c_{\delta}^{2}\right)$ denote the square of the radius of sphere $S$. Notice also that $x_{\delta+1}=F\left(x_{1}, \ldots, x_{\delta}\right)$ is the equation of a hyperparaboloid $\mathcal{H}$ in $(\delta+1)$-dimensional space, so that $F\left(x_{\delta+1,1}, \ldots, x_{\delta+1, \delta}\right)=F\left(p_{\delta+1}\right)$ is the signed height of the point obtained by lifting $p_{\delta+1}$ from the hyperplane $x_{\delta+1}=0$ (to which it belongs) to $\mathcal{H}$. Notice that hyperplane $x_{\delta+1}=0$ contains the hypersphere $S$.

We now wish to bound from above the probability of the event $\left|F\left(p_{\delta+1}\right)\right| \leq V$, for some constant $V$.

Assuming as usual constant density, this probability is the volume of a sphere $S^{\prime \prime}$ of radius $u^{\prime \prime}=\sqrt{V+u^{2}}$ minus the volume of a concentric sphere $S^{\prime}$ of radius $u^{\prime}=$ $\sqrt{-V+u^{2}}$, whenever the latter is defined. (These spheres are intersected with $\mathcal{C}_{\delta}$ and normalized by its volume.) In general dimension, since the above radii depend upon the points $p_{1}, p_{2}, \ldots, p_{\delta}$, the evaluations of the volumes of the above spheres is problematic. However, we shall show that an interesting simplification occurs for $\delta=2$.

Below we use the following bounding technique. Let $\Delta_{\delta}$ be expressed as the product of two continuous random variables

$$
\Delta_{\delta}=a b .
$$


Given a constant $\alpha$ we have

$$
\begin{aligned}
\operatorname{Prob}(a b \leq V) & \leq \operatorname{Prob}\left(a \leq \alpha \text { or } b \leq \frac{V}{\alpha}\right) \\
& \leq \operatorname{Prob}(a \leq \alpha)+\operatorname{Prob}\left(b \leq \frac{V}{\alpha}\right)-\operatorname{Prob}\left(a \leq \alpha \text { and } b \leq \frac{V}{\alpha}\right),
\end{aligned}
$$

that is,

$$
\operatorname{Prob}(a b \leq V) \leq \operatorname{Prob}(a \leq \alpha)+\operatorname{Prob}\left(b \leq \frac{V}{\alpha}\right)
$$

4.2.1. 2-Insphere Test. In this case the volumes of $S^{\prime \prime}$ and $S^{\prime}$ are respectively $\pi\left(V+u^{2}\right)$ and $\pi\left(-V+u^{2}\right)$, so that, if $S^{\prime}$ is defined, then their difference becomes $\pi 2 V$, otherwise (i.e., when $\left.u^{2} \leq V\right) \pi\left(V+u^{2}\right) \leq \pi 2 V$. After normalization (since 4 is the measure of the unit square) we have

$$
\operatorname{Prob}\left(\left|F\left(p_{3}\right)\right| \leq V\right) \leq \frac{\pi V}{2} .
$$

Given that $\left|\Delta_{2}\right|=\left|p_{1} p_{2}\right| F\left(p_{3}\right)$ inequality (e) becomes

$$
\begin{aligned}
\operatorname{Prob}\left(\left|\Delta_{2}\right| \leq V\right) & \leq \operatorname{Prob}\left(\left|p_{1} p_{2}\right| \leq \alpha\right)+\operatorname{Prob}\left(\left|F\left(p_{3}\right)\right| \leq \frac{V}{\alpha}\right) \\
& \leq \psi_{2} \alpha+\frac{\pi V}{2 \alpha} .
\end{aligned}
$$

From Section 3 we know that $\psi_{2}=\pi$. Selecting for $\alpha$ the critical value $\alpha=\sqrt{V / 2}$ we have

$$
\operatorname{Prob}\left(\left|\Delta_{2}\right| \leq V\right)=\pi \sqrt{2 V} \approx 4.44 \sqrt{V}
$$

4.2.2. $\delta$-Insphere Test $(\delta>2)$. As for the two-dimensional case, we prove that, in general, the probability that $\left|F\left(p_{\delta+1}\right)\right|$ is no larger than $W$ is sufficiently small.

Again, inequality (e) becomes

$$
\operatorname{Prob}\left(\left|\Delta_{\delta}\right| \leq W\right) \leq \operatorname{Prob}\left(\left|p_{1}, \ldots, p_{\delta}\right| \leq \frac{W}{\alpha}\right)+\operatorname{Prob}\left(\left|F\left(p_{\delta+1}\right)\right| \leq \alpha\right) .
$$

We know, from the results of Section 3, that

$$
\operatorname{Prob}\left(\left|p_{1}, \ldots, p_{\delta}\right| \leq \frac{W}{\alpha}\right) \leq \psi_{\delta} \frac{W}{\alpha} .
$$

Therefore, there remains to bound from above $\operatorname{Prob}\left(\left|F\left(p_{\delta+1}\right)\right| \leq \alpha\right)$. Recall that $\left|F\left(p_{\delta+1}\right)\right|$ is the distance from the plane $x_{\delta+1}=0$ of the point $p_{\delta+1}$ lifted to the hyperparaboloid $\mathcal{H}$, of equation $F(p)=0$, which intersects $x_{\delta+1}=0$ in a $\delta$-dimensional sphere passing by the origin with center $q=\left(c_{1} / 2, \ldots, c_{\delta} / 2\right)$. We define point $p_{\delta+1}^{*}$ such that it lies on a line $l$ passing by $O$ and $q$ and such that length $\left(p_{\delta+1}^{*}, q\right)=\operatorname{length}\left(p_{\delta+1}, q\right)$ and among the two possible choices, we select the one closest to $O$ (see Fig. 4). It is immediate that $\left|F\left(p_{\delta+1}\right)\right|=\left|F\left(p_{\delta+1}^{*}\right)\right|$ and that length $\left(O p_{\delta+1}^{*}\right) \leq \sqrt{\delta}$. 


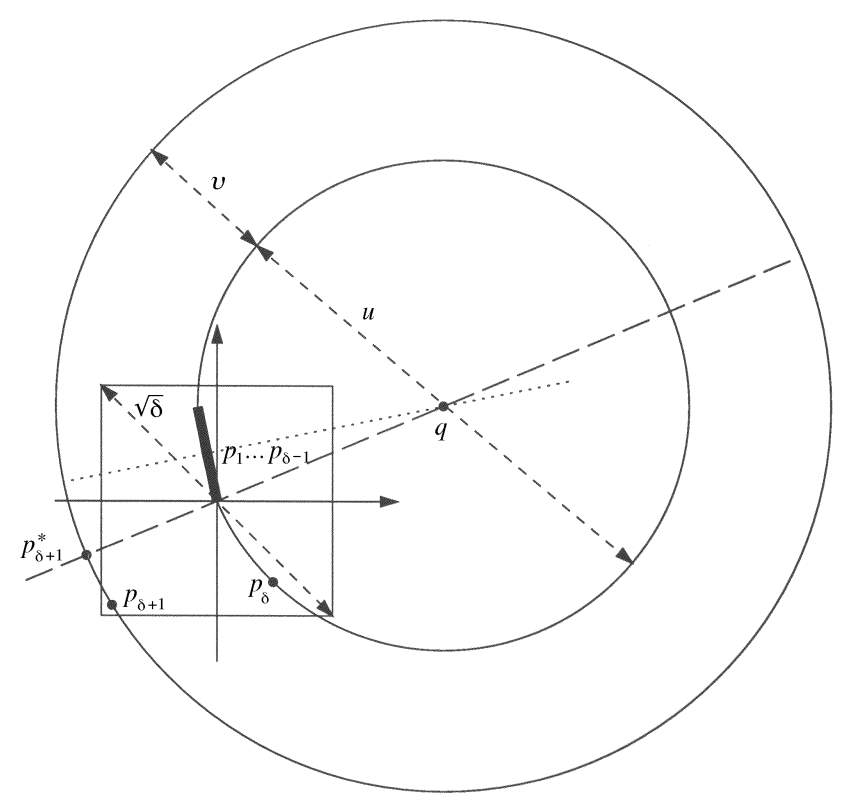

Fig. 4. Definition of $p_{\delta+1}^{*}, u$ and $v$, using a two-dimensional instantiation.

Thus, our problem is reduced to a one-dimensional instance, closely related to the one we studied in Section 4.1 (here $u=2$ length $(O q)$ and $v$ is the signed value of length $\left.\left(O p_{\delta+1}^{*}\right)\right)$. There are, however, some significant differences. There, we were evaluating the probability of the event $|u F(v)| \leq V$, and variables $u$ and $v$ were uniformly distributed in $[-1,1]$. Here, on the other hand, we wish to evaluate the probability of the event $|F(v)| \leq \alpha$, "radius" $u$ varies between 0 and $\infty$, and "distance" $v$ varies between $-\sqrt{\delta}$ and $\sqrt{\delta}$. Moreover, the densities $p_{1}(u)$ and $p_{2}(v)$ of $u$ and $v$ respectively are not constant; however, as we shall see, they are bounded by constants $q_{1}$ and $q_{2}$.

Next, we observe that

$$
\begin{aligned}
\operatorname{Prob}\left(\left|F\left(p_{\delta+1}\right)\right| \leq \alpha\right) & =\int_{u=0}^{\infty} \int_{v=-\sqrt{\delta}}^{\sqrt{\delta}} p_{1}(u) p_{2}(v) \operatorname{Prob}\left(\left|F\left(p_{\delta+1}\right)\right| \leq \alpha \mid u, v\right) d v d u \\
& =2 \int_{u=0}^{\infty} \int_{v=0}^{\sqrt{\delta}} p_{1}(u) p_{2}(v) \operatorname{Prob}\left(\left|F\left(p_{\delta+1}\right)\right| \leq \alpha \mid u, v\right) d v d u .
\end{aligned}
$$

Since the function $F\left(p_{\delta+1}\right)$ has the expression $v^{2}-u v$, the right-hand side of the above equation is just the integral of $p_{1}(u) p_{2}(v)$ on the domain (shown in Fig. 5) bounded by the curves $v^{2}-u v= \pm \alpha$. This integration domain can be split into four subdomains $\mathrm{A}$, $\mathrm{B}, \mathrm{C}, \mathrm{D}$, as shown in Fig. 5. This split depends on a parameter $\beta \leq \sqrt{\alpha}$ which will be chosen later. In detail we have

- Domain A is defined by $\beta \leq v \leq \sqrt{(} \delta)$ and $v-\alpha / v \leq u \leq v+\alpha / v \cdot p_{1}(u) p_{2}(v) \leq$ 


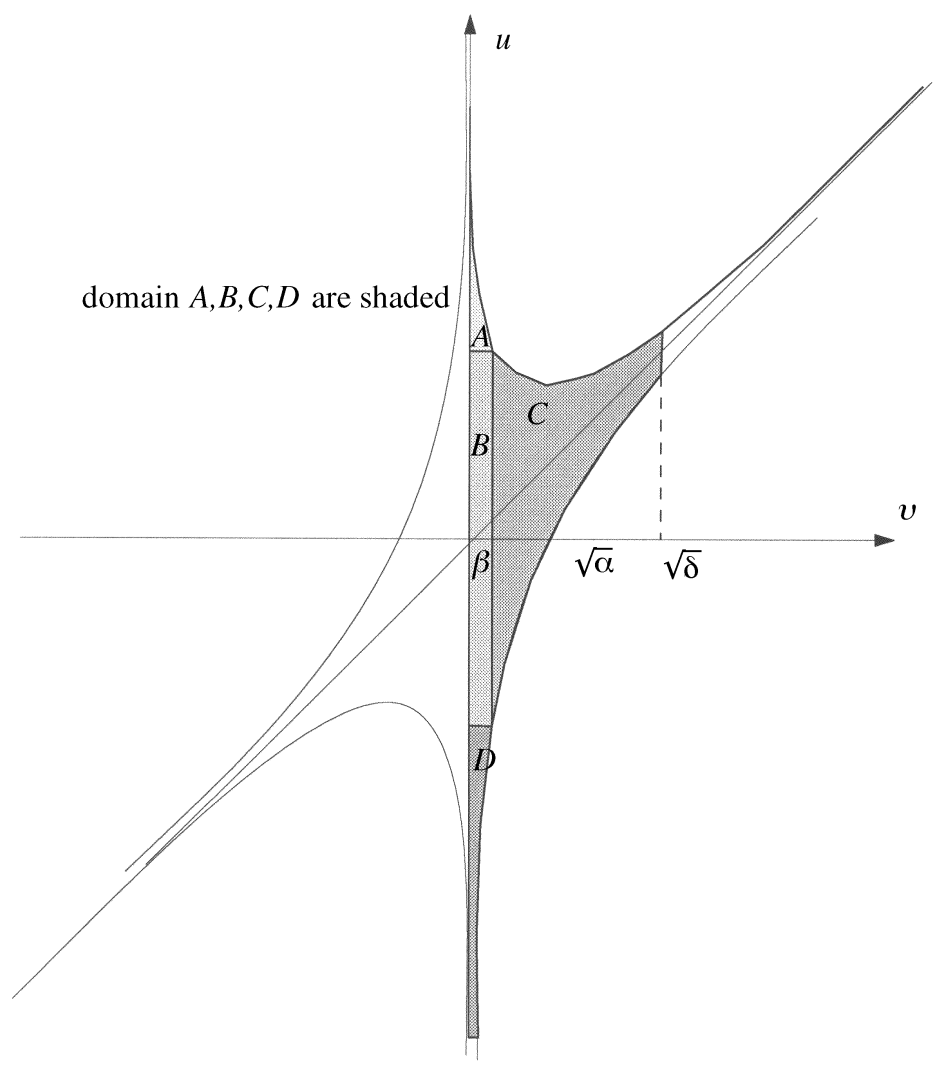

Fig. 5. Integration domain.

$q_{1} q_{2}$ and thus

$$
\iint_{A} p_{1}(u) p_{2}(v) d v d u \leq q_{1} q_{2} \int_{\beta}^{\sqrt{\delta}} \frac{2 \alpha}{v} d v \leq q_{1} q_{2}\left(\ln \delta+2 \ln \frac{1}{\beta}\right) \alpha .
$$

- Domain B is a rectangle defined by $0 \leq v \leq \beta$ and $\beta-\alpha / \beta \leq u \leq \beta+\alpha / \beta$. $p_{1}(u) p_{2}(v) \leq q_{1} q_{2}$ and thus

$$
\iint_{B} p_{1}(u) p_{2}(v) d v d u \leq q_{1} q_{2} \beta \frac{2 \alpha}{\beta} \leq 2 q_{1} q_{2} \alpha .
$$

- Domain $\mathrm{C}$ is defined by $0 \leq v \leq \beta$ and $\beta+\alpha / \beta \leq u \leq v+\alpha / v \cdot p_{2}(v) \leq q_{2}$ and since $\int p 1(u) d u \leq 1$ on any domain, we get

$$
\iint_{C} p_{1}(u) p_{2}(v) d v d u \leq q_{2} \beta .
$$




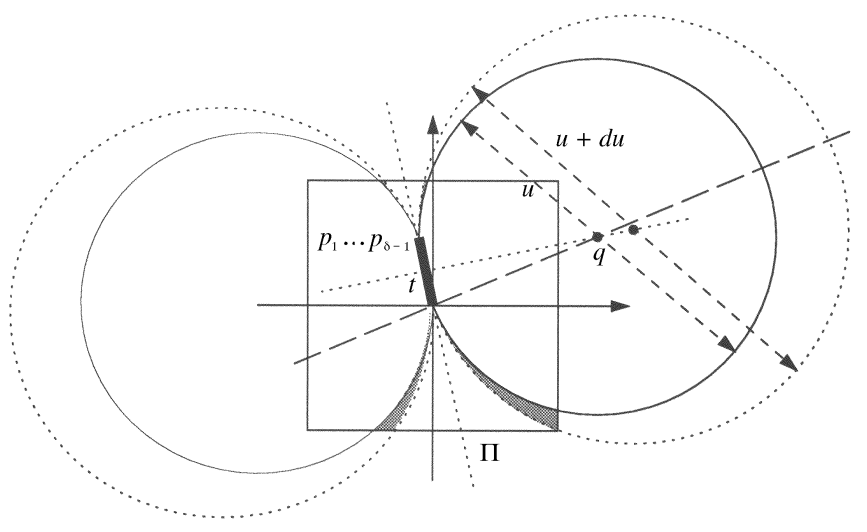

Fig. 6. For the analysis of $p_{1}(u)$.

- Domain D is analogous to domain C,

$$
\iint_{D} p_{1}(u) p_{2}(v) d v d u \leq q_{2} \beta .
$$

These results yield

$$
\operatorname{Prob}\left(\left|F\left(p_{\delta+1}\right)\right| \leq \alpha\right)=2 q_{1} q_{2} \alpha\left(\ln \delta+2 \ln \frac{1}{\beta}+2\right)+4 q_{2} \beta .
$$

To obtain values for $q_{1}$ and $q_{2}$, we observe:

1. With reference to $p_{1}(u)$, consider the $(\delta-1)$-dimensional sphere passing by $O, p_{1}, \ldots, p_{\delta-1}$ in the flat $\Pi$ defined by these points (see Fig. 6 for an illustration), and let $t$ be its center. Consider the family $\mathcal{F}$ of $\delta$-dimensional spheres passing through the origin and whose centers project to $t$ in $\Pi$. Point $p_{\delta}$ will determine a $\delta$-dimensional sphere of radius between $u$ and $u+d u$ if it lies between a pair of spheres of $\mathcal{F}$ (or its symmetric pair with respect to $\Pi$ ) of radii $u$ and $u+d u$. The volume $p_{1}(u) d u$ of this region (normalized by the volume $2^{\delta}$ of $\mathcal{C}_{\delta}$ ) is $\leq$ $\left(2 / 2^{\delta}\right) \mathcal{A}_{\delta} d u$, where $\mathcal{A}_{\delta}$ is the maximum of the measure of the surface of a $\delta$ dimensional sphere passing by the origin and intersected with $\mathcal{C}_{\delta}$. A trivial upper bound on $\mathcal{A}_{\delta}$ is the measure $2 \delta 2^{\delta-1}$ of the surface of $\mathcal{C}_{\delta}$. Therefore

$$
p_{1}(u) \leq \frac{2}{2^{\delta}} \cdot 2 \delta 2^{\delta-1}=2 \delta \triangleq q_{1} .
$$

2. With reference to $p_{2}(v)$, we observe that once $p_{\delta}$ is fixed, the choice of $p_{\delta+1}$ will produce a value between $v$ and $v+d v$ if $p_{\delta+1}$ belongs to the shaded region in Fig. 7. The volume of this region is $d v$ times the surface of the sphere of radius $v$ inside $\mathcal{C}_{\delta}$. This surface is clearly less than the surface of $\mathcal{C}_{\delta}$, so that we get

$$
p_{2}(v) \leq \frac{1}{2^{\delta}} \cdot 2 \delta 2^{\delta-1}=\delta \triangleq q_{2} .
$$




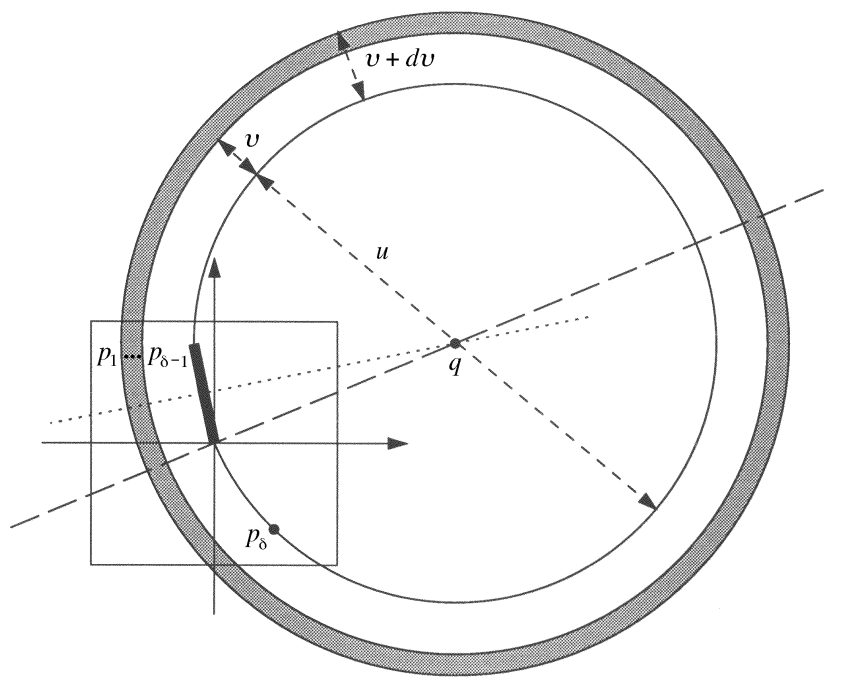

Fig. 7. For the analysis of $p_{2}(v)$.

Substituting $q_{1}=2 \delta$ and $q_{2}=\delta$ into (g) and choosing $\beta=\alpha / 2<\sqrt{\alpha}$ we obtain

$$
\begin{aligned}
\operatorname{Prob}\left(\left|F\left(p_{\delta+1}\right)\right| \leq \alpha\right) & \leq 8 \delta^{2} \alpha \ln \frac{1}{\alpha}+\left(4 \delta^{2} \ln \delta+8 \delta^{2} \ln 2+8 \delta^{2}+\delta\right) \alpha \\
& \triangleq \tau_{\delta} \alpha \ln \frac{1}{\alpha}+\theta_{\delta} \alpha
\end{aligned}
$$

(Small values of $\tau_{\delta}$ and $\theta_{\delta}$ are $\tau_{3}=72, \theta_{3}=164.5, \tau_{4}=128, \theta_{4}=309.4, \tau_{5}=200$, $\theta_{5}=504.6, \tau_{6}=288$, and $\theta_{6}=751.6$.) Inequality (f) becomes

$$
\operatorname{Prob}\left(\left|\Delta_{\delta}\right| \leq W\right) \leq \psi_{\delta} \frac{W}{\alpha}+\tau_{\delta} \alpha \ln \frac{1}{\alpha}+\theta_{\delta} \alpha
$$

so that, setting $\alpha=\sqrt{\psi_{\delta} W /\left(\tau_{\delta}+\theta_{\delta}\right)}$,

$$
\begin{aligned}
& \operatorname{Prob}\left(\left|\Delta_{\delta}\right| \leq W\right) \leq \sqrt{\psi_{\delta}\left(\tau_{\delta}+\theta_{\delta}\right)} \sqrt{W}\left(\ln \frac{1}{W}-\ln \frac{\tau_{\delta}+\theta_{\delta}}{\psi_{\delta}}+1\right) \\
& \triangleq \varphi_{\delta} \sqrt{W} \ln \frac{1}{W}+\chi_{\delta} \sqrt{W} \\
& \varphi_{3} \approx 70, \quad \chi_{3}=-100, \\
& \varphi_{4} \approx 408, \quad \chi_{4}=350, \\
& \varphi_{5} \approx 3970, \quad \chi_{5}=18,000, \\
& \varphi_{6} \approx 68,500, \quad \chi_{6}=640,000 \text {. }
\end{aligned}
$$




\subsection{Discrete Distribution}

As for the case of the which-side test, for the insphere test also we can map each point to the nearest grid point, and evaluate the ensuing effect on the determinant to be computed. Generalizing the notation of Section 3.2, we have that the norm $\left\|p_{i}^{\dagger}\right\|$ of the lifted point is bounded by $\sqrt{\delta+\delta^{2}}$ and that $\left\|d_{i}^{\dagger}\right\| \leq \sqrt{\delta} \sqrt{2\left(\eta^{2} / 4\right)+2(\eta / 2)} \leq(\sqrt{\delta} / \sqrt{2}) \sqrt{\eta+\eta^{2}}$. Therefore, grouping the errors term, we get

$$
\begin{aligned}
\left\|\mathcal{P}^{\dagger \dagger}|-| \mathcal{P}^{\dagger}\right\| & \leq\left[\sqrt{\delta+\delta^{2}}+\frac{\sqrt{\delta}}{\sqrt{2}} \sqrt{\eta+\eta^{2}}\right]^{\delta+1}-\sqrt{\delta+\delta^{2}} \delta+1 \\
& \lesssim(\delta+1){\sqrt{\delta+\delta^{2}}}^{\delta+1} \cdot \frac{\sqrt{\delta}}{\sqrt{2} \sqrt{\delta+\delta^{2}}} \sqrt{\eta+\eta^{2}}
\end{aligned}
$$

and, considering $\eta$ small,

$$
|| \mathcal{P}^{\prime \dagger}|-| \mathcal{P}^{\dagger}|| \lesssim(\delta+1) \sqrt{\frac{\delta}{2}} \sqrt{\delta+\delta^{2}} \delta \cdot \sqrt{\eta}
$$

\section{The Efficacy of Arithmetic Filters}

To complete the analysis, in this section we wish to assess, under the given statistical assumptions, the probability of failure of a given arithmetic filter for determinant sign evaluation (i.e., the probability that the filter is unable to certify the correctness of the computed sign). This probability is a quantitative measure of the efficacy of the filter. If $\left|p_{1}, \ldots, p_{\delta}\right|>\varepsilon_{\delta}$, then the result of the computation is reliable, and so is its sign. Plugging $\varepsilon_{\delta}$ in place of $V$ in the expression above, we obtain the condition

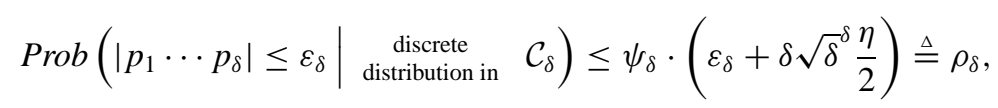

where we have used the result (Section 3) that $\operatorname{Prob}\left(\left|p_{1} \cdots p_{\delta}\right| \leq V\right) \leq \psi_{\delta} V$. The parameter $\rho_{\delta}$ introduced here is therefore the measure of filter efficacy sought.

To exemplify this approach, we compute $\rho_{\delta}$ for the evaluation of determinants, for the case where the coordinates of the points are floating-point numbers in the interval $[-1,1]$, the computations are carried out using floating-point arithmetic with $b$ bits of mantissa, and the determinant is evaluated by standard expansion with respect to one of its columns (recursive evaluation).

To this end, it is necessary to compute the parameter $\varepsilon_{\delta}$. We introduce the following notation:

- $\mathcal{E}[M, m]$ denotes the set of numbers whose absolute value is bounded by $M$ and whose error is bounded by $m$. Original entries belong to $\mathcal{E}[1,0]$.

- $\bar{M}$ denotes $2^{\lceil\log M\rceil}$. 
With this notation, if $x_{1} \in \mathcal{E}\left[M_{1}, m_{1}\right]$ and $x_{2} \in \mathcal{E}\left[M_{2}, m_{2}\right]$, then we have

$$
\begin{aligned}
x_{1}+x_{2} & \in \mathcal{E}\left[M_{1}+M_{2}, 2^{-b-1} \cdot \overline{M_{1}+M_{2}}+m_{1}+m_{2}\right], \\
x_{1} \cdot x_{2} & \in \mathcal{E}\left[M_{1} \cdot M_{2}, 2^{-b-1} \cdot \overline{M_{1} \cdot M_{2}}+m_{1} \cdot M_{2}+m_{2} \cdot M_{1}\right] .
\end{aligned}
$$

These rules express the mechanics of $b$-bit mantissa normalizing floating-point operations with round-off (round-off is done to the nearest, thus the error done is half of the value of the last bit). After transforming variable $y$ to an $\mathcal{E}[M, m]$ pair, we express the above rules as arithmetics on such pairs, as follows:

1. $\mathcal{E}\left[M_{1}, m_{1}\right]+\mathcal{E}\left[M_{2}, m_{2}\right]=\mathcal{E}\left[M_{1}+M_{2}, 2^{-b-1} \cdot \overline{M_{1}+M_{2}}+m_{1}+m_{2}\right]$.

2. $\mathcal{E}\left[M_{1}, m_{1}\right] \cdot \mathcal{E}\left[M_{2}, m_{2}\right]=\mathcal{E}\left[M_{1} \cdot M_{2}, 2^{-b-1} \cdot \overline{M_{1} \cdot M_{2}}+m_{1} \cdot M_{2}+m_{2} \cdot M_{1}\right]$.

The results given below are obtained in an Appendix to this paper by the mechanical application (with a few noted exceptions) of the above two rules to the recursive evaluation of a determinant. Using a $b$-bit mantissa, the results are:

$$
\begin{array}{lll}
\text { - } \varepsilon_{2}=2 \cdot 2^{-b}, & \bullet \varepsilon_{5}=576 \cdot 2^{-b}, & \bullet \varepsilon_{8}=226,624 \cdot 2^{-b} . \\
\text { - } \varepsilon_{3}=13 \cdot 2^{-b}, & \bullet \varepsilon_{6}=3672 \cdot 2^{-b}, \\
\text { - } \varepsilon_{4}=76 \cdot 2^{-b}, & \bullet \varepsilon_{7}=27,304 \cdot 2^{-b},
\end{array}
$$

For example, using the IEEE norm on $b=53$ bits, we can therefore estimate the corresponding probablility of failure. The pertinent values of $\varepsilon_{\delta}, \delta \sqrt{\delta}^{\delta}(\eta / 2)$, and $\rho_{\delta}$ are displayed below:

$$
\begin{array}{lll}
\bullet \varepsilon_{2}=2.2 \cdot 10^{-16}, & \bullet \delta \sqrt{\delta} \frac{\eta}{2}=1.6 \cdot 10^{-16}, & \bullet \rho_{2}=1.2 \cdot 10^{-15}, \\
\bullet \varepsilon_{3}=1.4 \cdot 10^{-15}, & \bullet \delta \sqrt{\delta} \frac{\eta}{2}=8.7 \cdot 10^{-16}, & \bullet \rho_{3}=4.8 \cdot 10^{-14}, \\
\bullet \varepsilon_{4}=8.4 \cdot 10^{-15}, & \bullet \delta \sqrt{\delta} \frac{\eta}{2}=7.1 \cdot 10^{-15}, & \bullet \rho_{4}=5.9 \cdot 10^{-12} \\
\bullet \varepsilon_{5}=5.7 \cdot 10^{-14}, & \bullet \delta \sqrt{\delta} \frac{\eta}{2}=7.8 \cdot 10^{-14}, & \bullet \rho_{5}=3.0 \cdot 10^{-9}, \\
\bullet \varepsilon_{6}=8.3 \cdot 10^{-13}, & \bullet \delta \sqrt{\delta} \frac{\eta}{2}=1.1 \cdot 10^{-12}, & \bullet \rho_{6}=8.7 \cdot 10^{-6} .
\end{array}
$$

Finally, it is important to evaluate the efficiency of the examined filter. We observe here that a recursive evaluation uses $\delta$ ! operations, but the cost can be reduced by using dynamic programming. In fact, the recursive evaluation involves $\left(\begin{array}{l}\delta \\ i\end{array}\right)$ minors of dimension $i$. In turn each such minor involves $(2 i-1)$ arithmetic operations ( $i$ multiplication and $i-1$ additions), whose operands are either minors of smaller dimension or original coefficients (for $i=2$, where the recursion stops). Thus, the total number of operations is

$$
r_{\delta}=\sum_{0}^{\delta-2}\left(\begin{array}{l}
\delta \\
i
\end{array}\right)(2(\delta-j)-1)=(\delta-1)\left(2^{\delta}-1\right) \sim \delta 2^{\delta} .
$$

For $\delta \leq 7$ we obtain $r_{1}=0, r_{2}=3, r_{3}=14, r_{4}=45, r_{5}=124, r_{6}=315, r_{7}=762$, and $r_{8}=1785$.

It must be pointed out that, for the same function considered above (determinant value), alternative evaluation schemes (corresponding to different expressions of the 
given function, such as other expansion rules, Gaussian elimination, etc.) and/or different arithmetic engines may be chosen. Each such choice would embody a filter, whose efficacy and efficiency can be assessed with the outlined method.

\section{Summary of Results and Conclusions}

In this paper we have developed a general approach to the assessment of the efficacy of arithmetic filters, under some reasonable probability assumptions. As an important example, we have considered the efficacy of filters for the evaluation of signs of determinants, both for a case where all entries are independent (the "which-side" predicate) and for a case where dependencies exist (the "insphere" predicate). This analysis, in general, consists of two parts.

The first part aims at computing the threshold for certification by the filter (i.e., the maximum error which can be generated by the evaluation process), and does not rest on any assumption about the distribution of the data. As an example, we have carried out this threshold analysis for the so-called recursive evaluation procedure, which computes a determinant by expanding it with respect to one of its columns.

The second part, which is considerably more subtle, aims at establishing the probability of failure of the filter, i.e., the probability that the result of the computation falls below the threshold. This analysis rests on a priori assumptions on the distribution of the input data, which we have taken as uniform within their representation range, and has been carried out for the two important geometric tests mentioned above. With the notations introduced in the preceding sections, the results are summarized below:

$$
\begin{aligned}
\operatorname{Prob}\left(\left|p_{1}, p_{2}, \ldots, p_{\delta}\right| \leq V \mid p_{i} \text { continuous in } \mathcal{B}_{\delta}\right) & \leq \sigma_{\delta} V \\
\operatorname{Prob}\left(\left|p_{1}, p_{2}, \ldots, p_{\delta}\right| \leq V \mid p_{i} \text { continuous in } \mathcal{C}_{\delta}\right) & \leq \psi_{\delta} V \\
\operatorname{Prob}\left(\left|p_{1}, p_{2}, \ldots, p_{\delta}\right| \leq V \mid p_{i} \in \eta \text {-grid in } \mathcal{C}_{\delta}\right) & \leq \psi_{\delta} \cdot\left(V+\alpha_{\delta} \eta\right), \\
\operatorname{Prob}\left(\left|\Delta_{1}\right| \leq W \mid p_{i} \text { continuous in } \mathcal{C}_{1}\right) & \leq 5.36 W^{2 / 3}, \\
\operatorname{Prob}\left(\left|\Delta_{\delta}\right| \leq W \mid p_{i} \text { continuous in } \mathcal{C}_{\delta}\right) & \leq \varphi_{\delta} \sqrt{W} \ln \frac{1}{W}+\chi_{\delta} \sqrt{W}, \\
\operatorname{Prob}\left(\left|\Delta_{i}\right| \leq W \mid p_{i} \in \eta \text {-grid in } \mathcal{C}_{\delta}\right) & \leq \varphi_{\delta} \sqrt{W+\beta_{\delta} \sqrt{\eta}} \ln \frac{1}{W+\beta_{\delta} \sqrt{\eta}} \\
& +\chi_{\delta} \sqrt{W+\beta_{\delta} \sqrt{\eta}} .
\end{aligned}
$$

Below we repeat for synoptic convenience the definitions of the relevant constants and a tabulation of their values for small $\delta$ :

$$
\begin{aligned}
& \sigma_{\delta}=\delta \frac{v_{\delta-1}(1)^{\delta}}{v_{\delta}(1)^{\delta-1}}, \\
& \psi_{\delta}=\frac{\delta v_{\delta}(1) v_{\delta-1}(1)^{\delta} \sqrt{\delta} \delta(\delta-1)}{2^{\delta^{2}}}, \\
& \varphi_{\delta}=\sqrt{\psi_{\delta}\left(2 \delta^{2} \ln \delta+4 \delta^{2} \ln 2+8 \delta^{2}+\frac{\delta}{2}\right)},
\end{aligned}
$$




$$
\begin{aligned}
& \chi_{\delta}=\varphi \delta\left(1-\ln \frac{2 \delta^{2} \ln \delta+4 \delta^{2} \ln 2+8 \delta^{2}+\delta / 2}{\psi_{\delta}}\right), \\
& \alpha_{\delta}=\frac{\delta \sqrt{\delta}^{\delta}}{2}, \\
& \beta_{\delta}=(\delta+1) \sqrt{\frac{\delta}{2}} \sqrt{\delta+\delta^{2}} \delta
\end{aligned}
$$

The values for small $\delta$ are

\begin{tabular}{rcccccr}
\hline$\delta$ & $\sigma_{\delta}$ & $\psi_{\delta}$ & $\varphi_{\delta}$ & $\chi_{\delta}$ & $\alpha_{\delta}$ & \multicolumn{1}{c}{$\beta_{\delta}$} \\
\hline 1 & 1 & 1 & & & 0.5 & 2 \\
2 & 2.5 & 3.2 & & 4.4 & 2 & 18 \\
3 & 5.3 & 21 & 70 & -100 & 7.8 & 200 \\
4 & 10 & 380 & 408 & 350 & 32 & 2,800 \\
5 & 19 & 23,000 & 3,970 & 18,000 & 140 & 47,000 \\
6 & 35 & $4.5 \cdot 10^{6}$ & 68,500 & 640,000 & 648 & 900,000 \\
\hline
\end{tabular}

The above values are tight only for the constant $\sigma$. Due to data interdependencies, the analysis of the insphere predicate is considerably more involved than that of the which-side predicate, and the adverse effect of the dependencies is manifest in the larger values of the probability of failure.

Such analysis is particularly valuable for estimating the time required to test the determinant sign. Under the given probability assumptions, we may conclude that for small dimension $(\leq 6)$ straightforward floating-point filters (i.e., floating-point evaluators) are extraordinarily effective.

\section{Appendix. Error Evaluation}

$D_{i}$ represents a generic determinant of dimension $i$, and $x$ a generic original coordinate. It must be pointed out that the recursive evaluation technique yields an upper bound of $\delta$ ! for $D_{\delta}$ because dependencies among data are not exploited. However, smaller values of such bound are known: since the determinant value is bounded by the product of the norms of its $\delta$ components, $\sqrt{\delta} \delta$ is an upper bound on $D_{\delta}$, which is attained when $\delta$ is a power of two (Hadamard matrices). For small values of $\delta$ the following bounds have been obtained (some by exhaustive calculation): $D_{1} \leq 1, D_{2} \leq 2, D_{3} \leq 4, D_{4} \leq 16$, $D_{5} \leq 48, D_{6} \leq 160, D_{7} \leq 576$ and $D_{8} \leq 4096$. Whenever applicable, we use these results below to obtain tighter estimates. These estimates have the form

$$
D_{\delta} \in \mathcal{E}\left[G_{\delta}, \varepsilon_{\delta}\right]
$$

where $\varepsilon_{\delta}$ is the object of the analysis and $G_{\delta}$, the largest value attainable by $D_{\delta}$, is only needed to carry out the analysis.

1. $D_{1} \in \mathcal{E}[1,0]$, by definition. 
2. $D_{2} \in \mathcal{E}\left[2,2^{-b+1}\right]$. In fact $D_{2}=x \cdot D_{1}+x \cdot D_{1}, x \in \mathcal{E}[1,0]$ and $D_{1} \in \mathcal{E}[1,0]$. Therefore

$$
\mathcal{E}[1,0] \cdot \mathcal{E}[1,0]+\mathcal{E}[1,0] \cdot \mathcal{E}[1,0]=\mathcal{E}\left[1,2^{-b-1}\right]+\mathcal{E}\left[1,2^{-b-1}\right]=\mathcal{E}\left[2,2^{-b+1}\right] .
$$

3. $D_{3} \in \mathcal{E}\left[4,13 \cdot 2^{-b}\right]$. In fact, $D_{3}=\left(x \cdot D_{2}+x \cdot D_{2}\right)+x \cdot D_{2}$. Therefore,

$$
\begin{aligned}
D_{3} \in & \left(\mathcal{E}[1,0] \cdot \mathcal{E}\left[2,2^{-b+1}\right]+\mathcal{E}[1,0] \cdot \mathcal{E}\left[2,2^{-b+1}\right]\right)+\mathcal{E}[1,0] \cdot \mathcal{E}\left[2,2^{-b+1}\right] \\
& =\mathcal{E}\left[4,2^{-b-1} \cdot 4+6 \cdot 2^{-b}\right]+\mathcal{E}\left[2,3 \cdot 2^{-b}\right] \\
& =\mathcal{E}\left[4,2^{-b-1} \cdot 4+11 \cdot 2^{-b}\right]=\mathcal{E}\left[4,13 \cdot 2^{-b}\right]
\end{aligned}
$$

where we have used the fact $D_{3} \leq 4$.

4. $D_{4} \in \mathcal{E}\left[16,19 \cdot 2^{-b+2}\right]$. The result is obtained by applying the previous rules and $x \in \mathcal{E}[1,0], D_{3} \in \mathcal{E}\left[4,13 \cdot 2^{-b}\right]$ to the following evaluation scheme:

$$
D_{4}=\left(x \cdot D_{3}+x \cdot D_{3}\right)+\left(x \cdot D_{3}+x \cdot D_{3}\right) .
$$

5. $D_{5} \in \mathcal{E}\left[48,129 \cdot 2^{-b+2}\right]$. The result is obtained by applying the previous rules and $x \in \mathcal{E}[1,0], D_{4} \in \mathcal{E}\left[16,19 \cdot 2^{-b+2}\right]$ to the following evaluation scheme:

$$
D_{5}=\left(\left(x \cdot D_{4}+x \cdot D_{4}\right)+x \cdot D_{4}\right)+\left(x \cdot D_{4}+x \cdot D_{4}\right),
$$

also making use of the fact $D_{5} \leq 48$.

6. $D_{6} \in \mathcal{E}\left[160,459 \cdot 2^{-b+3}\right]$. The result is obtained by applying the previous rules and $x \in \mathcal{E}[1,0], D_{5} \in \mathcal{E}\left[48,125 \cdot 2^{-b+2}\right]$ to the following evaluation scheme:

$$
D_{6}=\left(\left(\left(x \cdot D_{5}+x \cdot D_{5}\right)+\left(x \cdot D_{5}+x \cdot D_{5}\right)\right)+\left(x \cdot D_{5}+x \cdot D_{5}\right)\right),
$$

also making use of the fact $D_{6} \leq 160$.

7. $D_{7} \in \mathcal{E}\left[576,3413 \cdot 2^{-b+3}\right]$. The result is obtained by applying the previous rules and $x \in \mathcal{E}[1,0], D_{6} \in \mathcal{E}\left[160,459 \cdot 2^{-b+3}\right]$ to the following evaluation scheme:

$$
D_{7}=\left(\left(\left(x \cdot D_{6}+x \cdot D_{6}\right)+\left(x \cdot D_{6}+x \cdot D_{6}\right)\right)+\left(\left(x \cdot D_{6}+x \cdot D_{6}\right)+x \cdot D_{6}\right)\right) \text {, }
$$

also making use of the fact $D_{7} \leq 576$.

8. $D_{8} \in \mathcal{E}\left[4096,3541 \cdot 2^{-b+6}\right]$. Again, the result is obtained by applying the previous rules and $x \in \mathcal{E}[1,0], D_{7} \in \mathcal{E}\left[576,3413 \cdot 2^{-b+3}\right]$ to the following evaluation scheme:

$$
D_{8}=\left(\left(\left(x \cdot D_{7}+x \cdot D_{7}\right)+\left(x \cdot D_{7}+x \cdot D_{7}\right)\right)+\left(\left(x \cdot D_{7}+x \cdot D_{7}\right)+\left(x \cdot D_{7}+x \cdot D_{7}\right)\right)\right),
$$

also making use of the fact $D_{8} \leq 4096$.

Remark. If the original values do not belongs to $\mathcal{E}[1,0]$ but to $\mathcal{E}[1, \epsilon]$ and $\epsilon$ is small enough, then the final error on $D_{i}$ must be increased by $i ! \epsilon . \epsilon$ small enough means that the error on $M$ does not affect the value $\bar{M}$, in particular if all the manipulated sets are of the form $\mathcal{E}[M, m]$ with $\bar{M}=\overline{M+m}$, then adopting a first-order approximation is legitimate. 


\section{References}

[B] M. Berger. Geometry, (vols. 1-2). Springer-Verlag, New York, 1987.

$\left[\mathrm{BKM}^{+}\right]$C. Burnikel, J. Könnemann, K. Mehlhorn, S. Näher, S. Schirra, and C. Uhrig. Exact geometric computation in LEDA. In Proc. 11th Ann. ACM Symp. Comput. Geom., pages C18-C19, 1995.

[BMS] C. Burnikel, K. Mehlhorn, and S. Schirra. How to compute the Voronoi diagram of line segments: theoretical and experimental results. In Proc. 2nd Ann. European Symp. Algorithms, Lecture Notes in Computer Science, pages 227-239, vol. 855. Springer-Verlag, Berlin, 1994.

[FV] S. Fortune and C. J. Van Wyk. Efficient exact arithmetic for computational geometry. In Proc. 9th Ann. ACM Symp. Comput. Geom., pages 163-172, 1993.

[LPT] G. Liotta, F. P. Preparata, and R. Tamassia. Robust Proximity Queries in Implicit Voronoi Diagrams. Technical Report CS-96-16, Center for Geometric Computing, Computer Science Department, Brown University, Providence, RI, 1996.

[MN] K. Mehlhorn and S. Näher. The implementation of geometric algorithms. In Proc. 13th World Computer Congress IFIP94, volume 1, pages 223-231, 1994.

[Y] C. Yap. Towards exact geometric computation. Comput. Geom. Theory Appl., 7:3-23, 1997.

Received September 15, 1996, and in revised form September 9, 1997. 